Long-Term Population Decline

in Europe: The Relative Importance of Tempo Effects and Generational Length

Joshua Goldstein

Wolfgang Lutz

Sergei Scherbov

RR-04-004

March 2004 


\section{Long-Term Population Decline in Europe: The Relative Importance of Tempo Effects and Generational Length}

Joshua Goldstein

Princeton University, Princeton, NJ, USA

Wolfgang Lutz

International Institute for Applied Systems Analysis, Laxenburg, Austria

Sergei Scherbov

International Institute for Applied Systems Analysis, Laxenburg, Austria

RR-04-004

March 2004

Reprinted from Population and Development Review 29(4):699-707 (2003).

International Institute for Applied Systems Analysis - Schlossplatz 1 - A-2361 Laxenburg • Austria Tel: (+43 2236) 807 • Fax: (+43 2236) 71313 • E-mail: publications@iiasa.ac.at • Web: www.iiasa.ac.at 
Research Reports, which record research conducted at IIASA, are independently reviewed before publication. Views or opinions expressed herein do not necessarily represent those of the Institute, its National Member Organizations, or other organizations supporting the work.

Reprinted with permission from Population and Development Review 29(4):699-707 (December 2003). Copyright (c) Population and Development Review 2003.

All rights reserved. No part of this publication may be reproduced or transmitted in any form or by any means, electronic or mechanical, including photocopy, recording, or any information storage or retrieval system, without permission in writing from the copyright holder. 


\title{
Long-Term Population Decline in Europe: The Relative Importance of Tempo Effects and Generational Length
}

\author{
JOSHUA GOLDSTEIN \\ WOLFGANG LUTZ \\ SERGEI SCHERBov
}

IT HAS RECENTLY been suggested that ending the increase in the average age of childbearing in Europe would have a substantial effect on population dynamics by slowing population decline and aging. On the other hand, stable population theory suggests that under conditions of subreplacement fertility, longer generation length would imply slower shrinking, and thus produce a larger population in the long run. This note compares the relative importance of these two effects analytically and with actual data from the European Union.

In a recent contribution to the Policy Forum of Science entitled "Europe's population at a turning point," Lutz, O'Neill, and Scherbov (2003) showed that Europe has just entered a period of negative population momentuma tendency to decline in response to an old age structure, even if fertility were to instantly increase to replacement level. They tried to quantify the effect of continued increases in the mean age of childbearing (the so-called tempo effect) on the absolute number of births and future population dynamics in the 15-country European Union. They used as a baseline a scenario in which the tempo effect ends instantly-that is, the mean age of childbearing remains constant-and, as a result, the period total fertility rate (TFR) increases from 1.5 to 1.8 . This baseline was compared to a continuation of the tempo effect for 10, 20, 30, or 40 more years, which would keep the TFR lower by 0.3 over those periods. 
Lutz et al. found that the effects on population dynamics of continued low period fertility depressed by a continued increase in the mean age of childbearing are substantial. One method of calculation attributed 45 percent of future population decline to a continuation of the tempo effect until 2020. The consequences of continued increase in the mean age of birth on future population aging in the EU are also large. Seen from the perspective of the working-age population, defined as ages 15 to 64 , continued delay of 10 to 40 years would imply that, ceteris paribus, an additional 500 million to 1,500 million person-years of working-age persons would be needed to support the elderly population over the rest of the century, as compared with the no-delay scenario.

The demographic consequences of later childbearing have political implications. While governments in Europe are increasingly concerned about possible negative consequences of current low birth rates, there is also pronounced public resistance to any direct government interventions in the choice of one's family size, which is considered a private matter. In this context, policies that aim to influence the timing of births rather than family size might be more acceptable. Health benefits might provide an additional rationale for such policies, given that a continued delay in childbearing has led not only to burgeoning numbers of infertility treatments but also to increasing medical concerns about health risks for mothers and children associated with late pregnancies.

The conclusion of Lutz, O'Neill, and Scherbov that halting the trend toward higher mean ages of childbearing would significantly moderate population aging and decline of population size in Europe (over the rest of this century), however, seems to be in conflict with one of the implications of stable population theory. ${ }^{1}$ In a stable population with fertility below replacement level, a higher mean age of childbearing means a slower shrinking; a lower mean age of childbearing accelerates the process of shrinking through a faster succession of generations.

One would expect that the consequences of the tempo effect are more pronounced in the near future while those of a changing mean length of generation would only show up in the longer run. But this intuitive assessment does not make precise the consequences of possible policies aimed at influencing the mean age of childbearing. In this note, we quantify the effect on population size and aging of changing fertility timing, first through an analytical examination of this issue and then using numerical simulations applied to the current demographic situation of the European Union.

\section{An analytical answer}

Changes in the mean age of childbearing can influence the number of births through several distinct pathways. First, the tempo effect of advancing or 
postponing births has an immediate effect on the number of births in a calendar period (Bongaarts and Feeney 1998). Second, as suggested above, the generation-length effect will have consequences for the long-term population growth rate. Finally, changes in the timing of childbearing may influence the total fertility achieved by cohorts over their lifetime, with couples who start earlier having more children than couples who start late. This change in cohort fertility that accompanies changes in timing is known as the tempo-quantum interaction effect.

A model taking into account these three factors considers the number of births $B^{\star}(t)$ at some future time $t$ that result from a change in the mean age of childbearing relative to the number of births $B(t)$ that would have occurred had timing remained constant. Assuming the three factors are independent, then for $t$ long after fertility timing stops changing,

$$
\frac{B^{*}(t)}{B(t)}=\text { tempo effect } \times \text { generation-length effect }(t) \times \text { quantum effect }(t),
$$

where the quantum effect $(t)$ stands for the tempo-quantum interaction effect at time $t$. Note that the tempo effect is a one-time effect, whereas the generation-length and tempo-quantum interaction effects persist because they influence the underlying intrinsic rate of growth. In the case of a growing population, increasing the mean age of childbearing will depress all three factors. However, in the case of a shrinking population, later childbearing will depress future births through the tempo and tempo-quantum interaction effects, but inflate future births via the generation-length effect. Our goal is to explore which of these offsetting effects dominates, and when.

The relative magnitude of each of these three effects can be estimated fairly easily. For illustration, we consider the case of contemporary western Europe, where the cohort net reproduction rate (NRR) is roughly 0.9 and we take women's mean age of childbearing as 29 years. We investigate the consequences of a gradual increase in the mean age of childbearing to 33 years.

In the short term, higher ages of childbearing will reduce the number of births via the tempo effect. Even after the postponement of fertility comes to an end, the birth stream will continue to be smaller than it otherwise would have been, because the short-term decline in births will echo into subsequent generations. Goldstein and Schlag (1999) found that the longterm effect will be inversely proportional to the change in the mean age of childbearing. ${ }^{2}$ Specifically, an increase in the mean childbearing age from 29 to 33 years will reduce the long-term number of births by a factor of $1-29 / 33$, or about 12 percent, relative to what would have occurred had the childbearing timing remained constant.

The generation-length effect is also quantifiable. From stable population theory, the intrinsic growth rate is approximately $\log (\mathrm{NRR}) / \mu$, where the 
mean age of childbearing $\mu$ is used as an estimate of the mean length of a generation in the stable population. Taking derivatives with respect to $\mu$ gives $-\log (\mathrm{NRR}) / \mu^{2}$. Continuing our numerical example, an increase of one year in mean age will slow the rate of population decline by about $-\log (.9) / 29^{2}=$ $12 / 100,000$. A four-year increase slows the rate of decline from -0.36 percent to -0.32 percent. This is quite a small effect, less than $1 / 20$ th of one percent.

We can use these estimates of the tempo effect and generation length to ask how long it will take the generation-length effect, which influences the exponential growth rate over time, to overwhelm the one-time longterm effect of tempo. In our example, it will take about 250 years for the $1 / 20$ th of one percent change in the annual growth rate to offset the 12 percent change in the birth stream. For the next two or three centuries, however, the tempo effect will dominate.

Finally, we can consider the behavioral and biological effect of changes in women's timing on the total number of children born over women's lifetime. This is a difficult quantity to estimate, since changes in timing have also been accompanied by changes in fertility preferences. However, Kohler et al. (2001) have found that an increase of one year in the mean age of birth lowers cohort fertility by about 3 percent. Although such estimates are necessarily imprecise, their magnitude suggests that the total fertility effect will far outweigh any generation-length effect. In our example, increasing the mean age of childbearing from 29 to 33 years would reduce the NRR from 0.9 to about $0.9 *(0.97)^{4}=0.80$. Such a change in lifetime fertility would nearly double the rate of population decline from -0.36 percent to -0.68 percent, even after taking into account the moderating effect of longer generation length.

The analytical results suggest that the growth effects of changing generation length will be of minor importance. It will take several centuries for growth effects to reverse the initial tempo effect on births. Furthermore, the effect on growth rates is likely to be more than offset by changes in lifetime fertility.

It is possible to perform more exact analytical calculations by taking into account the full detail of the starting age structure. However, the complexities of such calculations make it convenient to turn to simulation to assess the effect of changes in fertility timing on population sizes in Europe. An added benefit of the simulations is that they allow one to see transient effects resulting from shifts in fertility rates in addition to the long-term impacts. They also allow us to look at changes in population age structure over time.

\section{A numerical answer through simulation}

The calculations presented below refer to the population of the European Union with its 15 member states as of 2003. They use the same baseline 
data in terms of the initial age distribution and fertility and mortality conditions as in the calculations by Lutz, O'Neill and Scherbov (2003) discussed above. As in that paper, we also consider a tempo effect of 0.3 in terms of TFR, which corresponds to an increase in the mean age of childbearing of 0.2 years per year or 2.0 years per decade starting with a mean age of 29 years in 2000 (Bongaarts 2002). Alternative calculations were performed for tempo effects lasting $0,10,20,30$, and 40 years. The figures below show only the cases of zero and 20 years of tempo effects, which clearly illustrate the main points.

The figures give three distinct scenarios:

Scenario 1 shows the case of an immediate end to the factor causing the tempo effect, that is, no further changes in the mean age of childbearing are assumed, hence period TFR is taken as constant at 1.8.

Scenario 2 shows the case in which the current tempo effect is assumed to continue for 20 more years without having an effect on total cohort fertility (i.e., no interactions). The period TFR is at 1.5 until 2020 when it increases to 1.8. In this case the curve of age-specific fertility rates is simply moved to the right by two years per decade. This also implies that birth rates above age 40 would increase considerably, which does not seem very realistic unless medically assisted conception becomes far more common than at present.

Scenario 3 assumes a continuation of the tempo effect as in Scenario 2 , but it also assumes that not all postponed births will be recuperated, hence there is a negative effect (tempo-quantum interaction) on total fertility. In this scenario we apply the simple method of proportionally reducing the age-specific fertility rates by 3 percent (the Kohler et al. estimate discussed above) for each year the age profile is shifted to the right, that is, to higher ages of childbearing.

We also applied more complex approaches that consider the nonlinearity of the tempo-quantum interaction attributable to the fact that at higher ages declining fecundability results in a greater reduction of fertility as women more closely approach a biological limit. In other words, an increase in the mean age of childbearing from 25 to 26 years should have a lower effect on the quantum than a shift from 31 to 32 . Since different approaches we applied also resulted in fertility reductions of around 3 percent per year for a shift starting around the current European mean age of 29 years, we decided to use the simpler linear assumption for the illustrative calculations in this note.

We emphasize that the three scenarios have been defined in order to analyze specific features of population dynamics and should not be mistaken for plausible or even likely future paths. The assumptions of a closed population with no further mortality changes and constant fertility after 2021 are certainly not plausible assumptions for the future of Europe, but 
they are useful for illustrating some important points. The projections were run for single-year age groups in single-year time steps.

Figure 1 gives the total population size of the current EU15 for these three scenarios over the next four centuries. Such a long time horizon is necessary in order to appropriately assess the long-term impacts of the different effects and compare them to the analytical reasoning given above. The figure clearly shows that over the first two centuries the positive effect of stopping the delay in childbearing (Scenario 1) clearly outweighs the effect of a longer mean length of generation in the case of 20 years of further delay (Scenario 2). Only during the twenty-third century does the generation-length effect catch up and only after 2300 does it dominate (in the case of no tempo-quantum interaction), but the difference between the two lines is much smaller than during the twenty-first century. Scenario 3, assuming a depressing effect of a higher mean age of childbearing on total fertility, lies far below the two other curves. This clearly confirms the view expressed in the analytical section that the presence of such a depressing tempo-quantum interaction effect completely dominates the picture, and the long-term positive effect of a longer generation becomes marginal.

Figure 2 gives the trends in old-age dependency ratios $(65+/ 15-64)$ generated by the same three scenarios. It extends only to 2150 because the stabilization of age structure under constant fertility and mortality condi-

FIGURE I Total population size of EU15 under three childbearing scenarios over four centuries

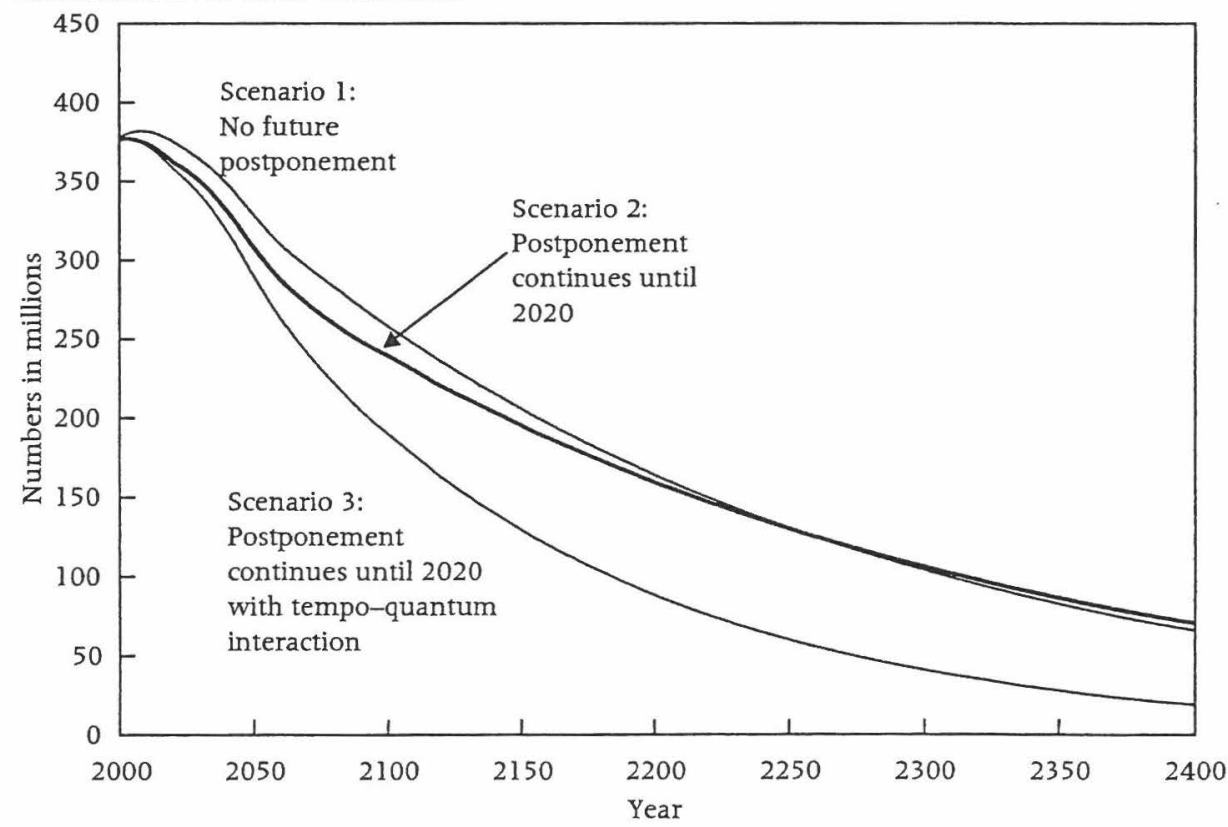

NOTE: Scenarios are illustrative, assuming no mortality decline and no migration. See text for details. 
FIGURE 2 Old-age dependency ratio of EU15 under three childbearing scenarios over one and a half centuries

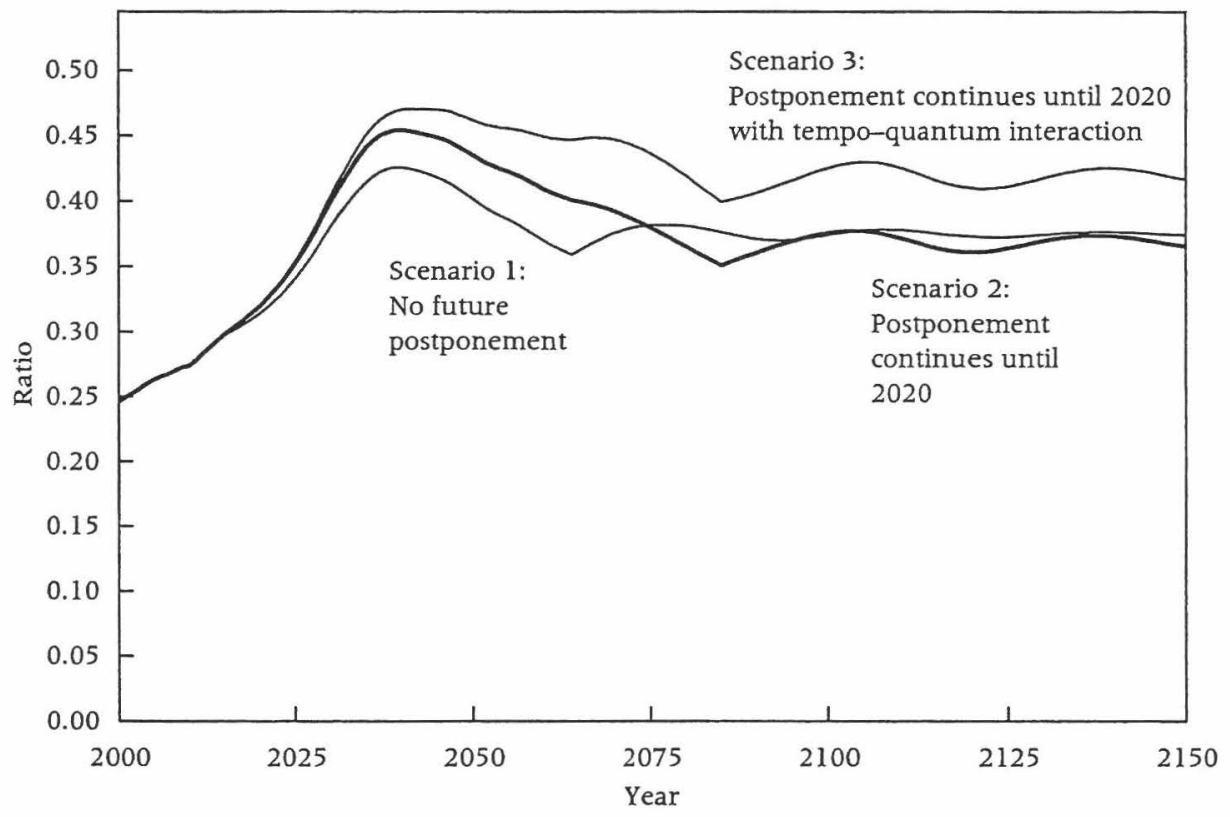

NOTE: Scenarios are illustrative, assuming no mortality decline and no migration. See text for details.

tions makes the picture less interesting in the longer run. This figure clearly confirms that with respect to population aging the tempo effect dominates the effect of longer generation length. A striking result is that for about half of the twenty-first century (from 2025 to 2075), the delay scenario (Scenario 2) has significantly higher dependency ratios than the no-delay scenario (Scenario 1), implying larger pension burdens for an extended period preceding the eventual convergence of the dependency ratios.

The comparison of Figures 1 and 2 also illustrates an important feature of stable population dynamics that is often forgotten by commentators on long-term population and environment trends. Because projections show that over the coming decades population aging in Europe will go hand in hand with population decline (unless large-scale immigration stops the decline), people unfamiliar with stable population theory tend to see these two trends as inseparable twins. On the global level one often hears the view that population aging (in the sense of an ongoing process toward an older and older population) is the price paid for limiting population growth (after an initial beneficial "demographic bonus"). But our long-term scenarios serve as a reminder that this is only true during a transitory period of some 80 years. After that, we could have a situation of further declining population sizethe dream of many ecologists - without having to pay the price of further aging, as distinct from an admittedly rather aged population. As Figure 2 shows, constant fertility even at low levels will result in a peak of the old age de- 
pendency burden around 2040 in Europe, still a consequence of the baby boom, to be followed by a moderate decline before stabilizing. This insight is clearly relevant for global climate change models that do have a time horizon of centuries, that see population size as a key driver of greenhouse gas emissions, and that consider population aging an obstacle to societies' capacities to adapt to unavoidable climate change.

\section{Conclusions}

In this short note we have demonstrated analytically and through a set of long-term population scenarios that a halt to the current tempo effect in Europe would have significant long-term moderating effects on population decline and aging. The objection that a continued postponement of childbearing also increases the generation length and therefore, under subreplacement fertility conditions, would also lead to slower population decline is theoretically correct but empirically unimportant for the next two centuries. Furthermore, it is likely that later childbearing will itself reduce total cohort fertility. Taking this effect into account overwhelms any effect of generation length.

We did not address the difficult question of what kinds of policy intervention might end further delays in childbearing or even decrease the mean age of childbearing over the coming decades. These issues will need further research and discussion. But this note prepares the demographic groundwork for such a discussion in demonstrating unambiguously that an end to further postponement of childbearing does have significant positive effects both on population size and on reducing the burden of population aging over the coming decades and in the long term. These benefits would be also enjoyed for a fairly long period into the future.

\section{Notes}

The order of authorship is alphabetical.

1 We thank Paul Demeny for bringing this point to our attention at the 2003 Annual Meeting of the Population Association of America. In the context of a discussion of European population trends he writes:

In the short and medium term, transition toward a lower mean generational length would be growth-promoting. But if fertility remains below replacement, the negative intrinsic growth rate would deplete the population more speedily, since the succeeding smaller and smaller generations would replace one another more quickly.
A higher generational length would, in the long run, stretch out the decline somewhat, moderating its annual tempo. (Demeny 2003: 3)

2 Goldstein and Schlag (1999) give the result for stationary populations. A full proof of the result for populations with non-zero growth rates is given in "Proof accompanying 'longer life and population growth,'" available at "http://opr.princeton.edu/ josh/ stretchproof.pdf $n$. The proof assumes that the intrinsic growth rate associated with cohort net fertility is constant. Standard results from renewal theory can also be used. 


\section{References}

Bongaarts, John. 2002. "The end of the fertility transition in the developed world," Population and Development Review 28(3): 419-443.

Bongaarts, John and Griffith Feeney. 1998. "On the quantum and tempo of fertility," Population and Development Review 24(2): 271-291.

Demeny, Paul. 2003. "Population policy dilemmas in Europe at the dawn of the twentyfirst century," Population and Development Review 29(1): 1-28.

Goldstein, Joshua R. and Wilhelm Schlag. 1999. "Longer life and population growth," Population and Development Review 25(4): 741-747.

Kohler, Hans-Peter, Axel Skytthe, and Kaare Christensen. 2001. "The age at first birth and completed fertility reconsidered: Findings from a sample of identical twins," MPIDR Working Paper WP 2001-006, Max Planck Institute for Demographic Research, Rostock, Germany. March. "http://www.demogr.mpg.de/Papers/Working/wp-2001-006.pdf».

Lutz, Wolfgang, Brian C. O'Neill, and Sergei Scherbov. 2003. "Europe's population at a turning point," Science 299: 1991-1992. 


\section{Ordering Information}

Orders must include the publication number and should be sent to the Publications Department, International Institute for Applied Systems Analysis, A-2361 Laxenburg, Austria.

Telephone: +432236807

Telefax: +432236 71313

E-mail: publications@iiasa.ac.at

A full list of IIASA publications is available at www.iiasa.ac.at/Publications 
International Institute for Applied Systems Analysis

Schlossplatz 1, A-2361 Laxenburg, Austria

$\rightarrow$ Tel: +432236 807 Fax: +43223671313

I I ASA www.iiasa.ac.at 